\title{
Comparison of vestibular rehabilitation on balance function in cochlear implant recipients
}

\begin{abstract}
Cochlear Implantation $(\mathrm{Cl})$ is an effective surgical approach to rehabilitate the deaf patients. However, the insertion of an electrode into the cochlea may have an adverse effect on vestibular receptors, resulting in vertigo or dizziness. The present study is aimed to investigate the impacts of vestibular rehabilitation therapy (VRT) exercises on dizziness symptoms of patients who underwent Cllimb.

Keywords: Tinnitus, Vestibular rehabilitation, Cochlear implant, Balance function
\end{abstract}




\section{INTRODUCTION}

Cochlear Implantation (Cl) picked up more noteworthy prominence as an advantageous technique for restoration of patients with serious to-significant sensorineural hearing misfortune $(\mathrm{SNHL}){ }^{1}$. By and large, $\mathrm{Cl}$ is a protected system with negligible danger of postoperative issues. In any case, the vestibular framework is anatomically and phylogenetically associated with the cochlea from the end organ to the focal pathways ${ }^{2}$. At that point, embeddings the $\mathrm{Cl}$ cathodes into the intra-cochlear liquid may meddle with the vestibular structures and lead to vestibular brokenness, for example, discombobulation and equilibrium issues ${ }^{3}$. Notwithstanding immediate injury to the vestibular framework, electrical incitement of the vestibular organs by the embedded cathodes can impact the equilibrium capacity of $\mathrm{Cl}$ beneficiaries. Wooziness or vertigo are normal difficulty following $\mathrm{Cl}$, and its occurrence changes broadly from $2 \%$ to $75 \%$. The sorts of wooziness announced in the $\mathrm{Cl}$ writing shift broadly from a continuous feeling of instability or unsteadiness to quick, intense assaults of vertigo. There are different helpful techniques to treat tipsiness indications, including pharmacological, careful, and vestibular restoration strategies. Among these methodologies, vestibular recovery treatment (VRT) is currently generally utilized as a non-obtrusive strategy in the administration of patients with fringe or focal vestibular problems. Past examinations have demonstrated that VRT projects could quicken the normal cycle of focal vestibular remuneration, lead to the disposal of indications and prior re-visitation of the standard way of life exercises. These activities likewise diminish the danger of falls, which can have problematic or now and then deadly outcomes in individuals of more seasoned age $\mathrm{e}^{4-6}$.

\section{METHODS}

A total of 21 patients (age range: 28 to 61 years) who experienced vertigo and dizziness following unilateral $\mathrm{Cl}$ were included. The etiology of deafness was idiopathic $(n=11)$, meningitis $(n=3)$, head trauma $(n=2)$, autoimmune inner ear disease $(n=1)$, Meniere's disease $(n=1)$ and chronic otitis media $(n=3)$. Cl operation was conducted in $62 \%$ of patients $(n=13)$ in the right ear. For all patients, $\mathrm{Cl}$ electrodes were inserted via a cochleostomy performed anterior to the round window niche. During the operation, no steroid drugs were administered via intravenous, intra-tympanic or intracochlear methods. All electrodes were fully inserted in the cochlea and after surgery, insertion of the electrodes array was confirmed by X-ray imaging. Patients with a history of central nervous system disease or orthopedic disorders were excluded. Those patients with inner ear anomalies determined on temporal bone computed tomography (CT) and magnetic resonance imaging (MRI) were also excluded. The protocols and all experimental procedures of the study were approved by the ethics committee of the local research committee, which were in complete agreement with the ethical regulations of human studies set by the Helsinki declaration (2013). After the enrolment of subjects and before the beginning of the investigation, objectives and protocols of the study and possible advantages and disadvantages of the treatments were clearly explained to all cases, and then, the participants filled and signed a written consent form on their cooperation in the study ${ }^{7}$. After taking a close account, and playacting a physical and proprioception perform assessments, AN exercise set up was developed for every patient. The VRT intervention was tailored to every patient's impairments that associated with symptom, nervus oculomotorius perform, and gait and balance performance. The medical aid set up consisted of a habituation (compensatory) and adaptation exercises together with gait and balance exercises. Habituation exercises utilize repetitive movement's stimuli.

\section{RESULTS}

For the primary outcome parameter, DHI, we found that total $\mathrm{DHI}$ score was improved about 16 points after 2-week and 23 points after 4-week vestibular rehabilitation exercises (Table 1). A repeated measure ANOVA revealed a significant reduction and a consequent improvement in $\mathrm{DHI}$ scores after vestibular exercises in all emotional, physical and functional domains $(p<0.001)$. Our results also demonstrated that the effect of implanted ear $(p=0.076)$ and gender $(p=0.094)$ factors on DHI scores

Table 1: Comparison of severity of disability $\mathrm{DHI}$ before and after the exercise.

\begin{tabular}{|c|c|c|c|}
\hline Severity of disability & Before intervention & After intervention & P-value \\
\hline Normal & 0 & 10 & \multirow{4}{*}{$X^{2}=9.16, p$-value $=0.01$} \\
\hline Mild & 8 & 7 & \\
\hline Moderate & 9 & 4 & \\
\hline Severe & 4 & 0 & \\
\hline
\end{tabular}

Table 2: Comparison of the mean dizziness handicap inventory (DHI) scores at different time points of study. Vestibular rehabilitation phase

$\begin{array}{cc}\text { Parameter } & \text { Pre-VRT } \\ \text { DHI-physical } & 15.14( \pm 3.43) \\ \text { DHI-emotional } & 16.86( \pm 3.97) \\ \text { DHI-functional } & 19.05( \pm 3.50) \\ \text { DHI-total } & 53.87( \pm 8.53)\end{array}$

Post 2-week VRT
$11.62( \pm 2.33)$
$11.24( \pm 2.40)$
$14.38( \pm 3.07)$
$38.26( \pm 5.51)$

Post 2-week VRT

Post 4-week VRT

$7.43( \pm 2.67)$

$5.90( \pm 1.94)$

$9.14( \pm 2.76)$

$30.06( \pm 4.43)$ 
were not statistically significant With respect to the DHI score, $61 \%(13 / 21)$ of patients reported moderate or severe degree of vestibular impairment at the initial visit. However, at the end of the therapy course only 4 patients (19.05\%) showed moderate or severe degree of impairment (Table 2).

The mean VAS score was $5.87 \pm 2.27$ at the initial testing and it decreased significantly during the course of the vestibular rehabilitation therapy (second week, $2.02 \pm 1.75, p<0.001$, and forth week, $1.51 \pm 1.29, p$ $<0.001)$. With respect to VAS score, the treatment was noted in 16 patients (76.2\%), and no change in 5 patients $(23.8 \%)^{8}$.

\section{DISCUSSION}

$\mathrm{Cl}$ is a common treatment option for patients with severe to profound SNHL. Although $\mathrm{Cl}$ is considered to be surgically a safe procedure, damage of vestibular function following operation has been noted in recent years. The recent evidence has demonstrated that $\mathrm{Cl}$ may cause trauma through the insertion of the electrodes into the inner ear, which may lead to an intraoperative loss of perilymph, labyrinthitis, perilymph fistula, and/or endolymphatic hydrops. It has been also indicated that the electrode insertion during the surgery may induce the osseous spiral lamina, basilar membrane, and vestibular end-organ lesions. Recent evidence has demonstrated that dizziness after $\mathrm{Cl}$ occurs quite frequently, with a reported incidence among series of between $2 \%$ and $60 \%$. The types of dizziness reported in the $\mathrm{Cl}$ literature vary widely from a gradual sense of unsteadiness or lightheadedness to immediate, acute attacks of vertigo. VRT is an exercised-based designed approach to enhance central nervous system compensation for vestibular disorder. These exercises are supposed to accelerate and improve central compensation via the mechanisms of habituation training, which maximize adaptation of the vestibulo-spinal reflexes (VSR) and vestibulo-ocular reflex (VOR), as well as increase substitution $^{9}$. In this study, following two weeks of supervised exercise program, a fast recovery was found in $\mathrm{Cl}$ patients. We also found that a significant reduction and a consequent improvement in VAS and total $\mathrm{DHI}$ scores between the second week and end of the treatment. Our study demonstrated that nearly $84 \%$ of the $\mathrm{Cl}$ recipients have showed improvement with respect to both VAS and DHI scores.

\section{CONCLUSION}

The results of the current study suggest that vestibular rehabilitation has a positive effect on the symptoms of the patients who underwent $\mathrm{Cl}$. These exercises lead to an improvement in balance and postural stability, and a reduction in the self-report measure of handicaps.

\section{CONFLICT OF INTEREST}

There are no conflicts of interest in terms of the present study.

\section{REFERENCES}

1. Patel TA, Nguyen SA, White DR. Clinical indicators of admission for pediatric cochlear implant procedures. Ann Otol Rhinol Laryngol. 2018; 127(7): 470-474.

2. Saki N, Bayat $A$, Nikakhlagh $S$, Karimi $M$, Nikafrooz $M$, Daneshi A, et al. A National Iranian Cochlear Implant Registry (ICIR): cochlear implanted recipient observational study. Int Tinnitus J. 2019; 23(2): 74-78.

3. Abouzayd M, Smith PF, Moreau S, Hitier M. What vestibular tests to choose in symptomatic patients after a cochlear implant? A systematic review and meta-analysis. Eur Arch Otorhinolaryngol. 2017; 274(1):53-63.

4. Manrique-Huarte R, Calavia D, Alvarez-Gomez L, Huarte A, Perez-Fernández $N$, Manrique $M$. Vestibulo-cochlear function after cochlear implantation in patients with Meniere's Disease. J Int Adv Otol. 2018; 14(1): 18-21.

5. Cushing SL, Gordon KA, Rutka JA, James AL, Papsin BC. Vestibular endo-organ dysfunction in children with sensorineural hearing loss and cochlear implants: an expanded cohort and etiologic assessment. Otol Neurotol. 2013; 34: 422-428.

6. Ajalloueyan M, Saeedi M, Sadeghi M, Zamiri F. The effects of cochlear implantation on vestibular function in 1-4 years old children. Int J Pediatr Otorhinolaryngol. 2017; 94: 100-103.

7. Miwa $T$, Minoda R, Matsuyoshi $H$, Takeda $H$. The effect of cochlear implants on vestibular-evoked myogenic potential responses and postural stability. Auris Nasus Larynx. 2019; 46(1): 50-57.

8. Krause E, Wechtenbruch J, Rader T, Gürkov R. Influence of cochlear implantation on sacculus function. Otolaryngol Head Neck Surg. 2009; 140(1): 108-113.

9. Tait M, Nikolopoulos TP, De Raeve L, Johnson S, Datta G, Karltorp E, et al. Bilateral versus unilateral cochlear implantation in young children. Int J Pediatr Otorhinolaryngol. 2010; 74: 206-211. 